\title{
New classes of Schrödinger equations equivalent to the free particle equation through non-local transformations
}

\author{
George Bluman and Vladimir Shtelen \\ Department of Mathematics, University of British Columbia, Vancouver, BC, Canada, V6T 1Z2
}

Received 22 August 1995, in final form 21 March 1996

\begin{abstract}
We introduce new classes of Schrödinger equations with time-dependent potentials which are transformable to the free particle equation through non-local transformations. These non-local transformations arise when considering the potential systems of the Schrödinger equation. Explicit formulae are given for the potentials and the corresponding solutions related to the solutions of the free particle equation.
\end{abstract}

\section{Introduction}

In this paper we extend previous work on finding Schrödinger equations which can be mapped into the free particle equation. Niederer (1973) showed that the Lie group of point transformations admitted by the Schrödinger equation for the harmonic oscillator is isomorphic to the Lie group of point transformations admitted by the free particle equation and exhibited a point transformation which related the solutions of these equations. Boyer (1974) extended Niederer's work to show that a Schrödinger equation with a timeindependent potential can be mapped by a point transformation into the free particle equation if and only if the potential is a quadratic polynomial. Bluman $(1980,1983)$ extended Boyer's work to time-dependent potentials by essentially showing that such Schrödinger equations can be mapped by point transformations to the free particle equation if and only if the potential is a quadratic polynomial with arbitrary time-dependent coefficients.

In the present paper we enlarge the previously known class of Schrödinger equations with time-dependent potentials which can be mapped into the free particle equation through the use of non-local transformations. We accomplish this by embedding a given Schrödinger equation in an auxiliary system of partial differential equations (PDEs) so that the set of all solutions of the auxiliary system yields all solutions of the given Schrödinger equation but there is not a one-to-one correspondence between the solutions of the given Schrödinger equation and those of related auxiliary systems. Such auxiliary systems of PDEs are 'potential' systems obtained through replacement of the given Schrödinger equation by equivalent conservation laws yielded by factors which are arbitrary solutions of the complex conjugate equation of the given Schrödinger equation (Bluman and Doran-Wu 1995). A point transformation of the variables of such a potential system, which maps the potential system to the corresponding potential system for a free particle equation, could yield a non-local transformation which maps the given Schrödinger equation to the free particle equation.

We find the most general class of time-dependent potentials for which point transformations map the corresponding potential systems into the free particle system. Such 
point transformations will include transformations which induce non-local transformations of the given Schrödinger equation. Consequently we obtain new classes of time-dependent potentials (and corresponding mappings of solutions) for which the Schrödinger equation can be mapped into the free particle equation. Our non-local transformations reduce to the known point transformations if and only if the potential is quadratic.

Our mapping formulae can be recycled to further enlarge the classes of Schrödinger equations which can be mapped into the free particle equation. The approach presented in this paper can be extended to other mapping problems. For example, new classes of diffusion equations transformable to the heat equation by non-local transformations are found in Bluman and Shtelen (1996).

\section{The basic framework}

Consider the Schrödinger equation

$$
\mathrm{i} \frac{\partial u_{1}}{\partial t}+\frac{\partial^{2} u_{1}}{\partial x^{2}}-V_{1}(x, t) u_{1}=0
$$

where $u_{1}(x, t)$ is a complex-valued function of the real variables $(x, t)$ and $V_{1}(x, t)$ is a real potential.

The most general point transformation of the form

$$
\tau=T\left(x, t, u_{1}\right) \quad y=Y\left(x, t, u_{1}\right) \quad u_{2}=F\left(x, t, u_{1}\right)
$$

which maps (2.1) into an equivalent equation of the same form, namely,

$$
\mathrm{i} \frac{\partial u_{2}}{\partial \tau}+\frac{\partial^{2} u_{2}}{\partial y^{2}}-V_{2}(y, \tau) u_{2}=0
$$

is given by

$$
\begin{aligned}
& y=\sigma(t) x+\rho(t) \quad \tau=\int^{t} \sigma^{2}(\mu) \mathrm{d} \mu \\
& u_{2}=\exp \left[\mathrm{i}\left(\frac{\dot{\sigma}}{4 \sigma} x^{2}+\frac{\dot{\rho}}{2 \sigma} x+\lambda(t)\right)\right] u_{1}
\end{aligned}
$$

with

$V_{2}(y, \tau)=\frac{1}{\sigma^{2}}\left[V_{1}(x, t)+\left(\frac{2 \dot{\sigma}^{2}-\sigma \ddot{\sigma}}{4 \sigma^{2}}\right) x^{2}+\left(\frac{2 \dot{\sigma} \dot{\rho}-\sigma \ddot{\rho}}{2 \sigma^{2}}\right) x+\left(\frac{\mathrm{i} \dot{\sigma}}{2 \sigma}+\frac{\dot{\rho}^{2}}{4 \sigma^{2}}-\dot{\lambda}\right)\right]$

where $\sigma(t), \rho(t), \lambda(t)$ are arbitrary functions of $t$ and $\dot{\sigma}=\mathrm{d} \sigma / \mathrm{d} t$, etc. Consequently, with respect to point transformations, (2.1) is equivalent to the free particle equation if and only if

$$
V_{1}(x, t)=\alpha(t) x^{2}+\beta(t) x+\gamma(t)
$$

for arbitrary $\alpha(t), \beta(t)$ and $\gamma(t)$.

Given a linear operator $\mathcal{L}$, its adjoint $\mathcal{L}^{*}$ is defined by

$$
\Phi \mathcal{L} u-u \mathcal{L}^{*} \Phi=\sum_{i=1}^{n} D_{i} f^{i}
$$

where $x=\left(x_{1}, x_{2}, \ldots, x_{n}\right)$, the total derivative operators $D_{i}=\partial / \partial x_{i}, i=1,2, \ldots, n$, and $\left\{f^{i}\right\}$ are bilinear expressions in $u, \Phi$ and their derivatives. Consequently, if

$$
\mathcal{L}^{*} \Phi=0
$$


then $\mathcal{L} u=0$ if and only if $\sum_{i=1}^{n} D_{i} f^{i}=0$, i.e. a given linear partial differential equation

$$
\mathcal{L} u=0
$$

is equivalent to the conservation law

$$
\sum_{i=1}^{n} D_{i} f^{i}=0
$$

for any $\Phi$ satisfying its adjoint equation (2.7).

We now specialize to the case when (2.8) is the Schrödinger equation (2.1). Here the linear operator is

$$
\mathcal{L}=\mathrm{i} \frac{\partial}{\partial t}+\frac{\partial^{2}}{\partial x^{2}}-V_{1}(x, t)
$$

its adjoint is given by

$$
\mathcal{L}^{*}=-\mathrm{i} \frac{\partial}{\partial t}+\frac{\partial^{2}}{\partial x^{2}}-V_{1}(x, t)
$$

and (2.9) becomes

$$
\frac{\partial}{\partial t}\left(\mathrm{i} \Phi u_{1}\right)+\frac{\partial}{\partial x}\left(\Phi \frac{\partial u_{1}}{\partial x}-\frac{\partial \Phi}{\partial x} u_{1}\right)=0 .
$$

The potential system corresponding to (2.12) is given by

$$
\begin{aligned}
& \frac{\partial v_{1}}{\partial x}=\mathrm{i} \Phi u_{1} \\
& \frac{\partial v_{1}}{\partial t}=\frac{\partial \Phi}{\partial x} u_{1}-\Phi \frac{\partial u_{1}}{\partial x}
\end{aligned}
$$

where $\Phi(x, t)$ is a solution of

$$
\mathcal{L}^{*} \Phi=-\mathrm{i} \frac{\partial \Phi}{\partial t}+\frac{\partial^{2} \Phi}{\partial x^{2}}-V_{1}(x, t) \Phi=0 .
$$

One observes that equation (2.14) is the complex conjugate of equation (2.1) if the potential $V_{1}(x, t)$ is real.

Note that if $\left(u_{1}(x, t), v_{1}(x, t), \Phi(x, t)\right)$ solves (2.13) and (2.14) then $u_{1}(x, t)$ solves the Schrödinger equation (2.1) and $v_{1}(x, t)$ solves

$$
\mathrm{i} \frac{\partial v_{1}}{\partial t}+\frac{\partial^{2} v_{1}}{\partial x^{2}}-\frac{2}{\Phi} \frac{\partial \Phi}{\partial x} \frac{\partial v_{1}}{\partial x}=0 .
$$

If $u_{1}(x, t)$ solves (2.1) and $\Phi(x, t)$ solves (2.14), then one can find $v_{1}(x, t)$ solving (2.13), i.e. for any $\Phi(x, t)$ satisfying (2.14), equations (2.1) and (2.15) are equivalent through the non-local transformation defined by (2.13).

For any $\Phi(x, t)$ satisfying (2.14), the point transformation

$$
w=\frac{v_{1}}{\Phi}
$$

maps (2.15) to

$$
\mathrm{i} \frac{\partial w}{\partial t}+\frac{\partial^{2} w}{\partial x^{2}}-V_{2}(x, t) w=0
$$

where the new potential $V_{2}(x, t)$ is given by

$$
V_{2}(x, t)=V_{1}(x, t)-2 \frac{\partial^{2}}{\partial x^{2}} \log \Phi .
$$


Equation (2.18) is known to connect the Schrödinger equation and the nonlinear $\mathrm{KdV}$ equation. In particular if $V_{1}(x, t)$ is a solution of the $\mathrm{KdV}$ equation and $\Psi(x, t)=1 / \Phi(x, t)$ solves the time-independent Schrödinger equation with potential $V_{1}(x, t)$, with $t$ treated as a parameter, then $V_{2}(x, t)$ also solves the KdV equation (see Dodd et al 1982, ch 3, and references therein).

From equations (2.13) and (2.16) it follows immediately that if $u_{1}(x, t)$ solves (2.1) then

$$
w=u_{2}(x, t)=\frac{\mathrm{i}}{\Phi(x, t)}\left[\int_{k}^{x} u_{1}(\xi, t) \Phi(\xi, t) \mathrm{d} \xi+B_{2}(t)\right]
$$

with $B_{2}(t)$ satisfying the condition

$$
\frac{\mathrm{d} B_{2}}{\mathrm{~d} t}=\mathrm{i}\left[\frac{\partial \Phi}{\partial x}(k, t) u_{1}(k, t)-\Phi(k, t) \frac{\partial u_{1}}{\partial x}(k, t)\right]
$$

for any constant $k$, solves the Schrödinger equation (2.17). (Note that if both $\Phi(x, t)$ and $u_{1}(x, t)$ satisfy the same homogeneous boundary condition at $x=k=k^{*}$, then $B_{2}=0$ satisfies condition (2.19b). Moreover, it is easy to see that if $\Phi\left(k^{*}, t\right)=u_{1}\left(k^{*}, t\right)=0$, then $u_{2}\left(k^{*}, t\right)=0$.) Conversely, if $w(x, t)$ solves (2.17) and $\phi=\log \Phi$ is any particular solution of

$$
\mathrm{i} \frac{\partial \phi}{\partial t}+\frac{\partial^{2} \phi}{\partial x^{2}}-\left(\frac{\partial \phi}{\partial x}\right)^{2}=-V_{2}(x, t)
$$

then solving (2.14) in terms of $V_{1}(x, t)$, i.e. setting

$$
V_{1}(x, t)=-\mathrm{i} \frac{\partial \phi}{\partial t}+\frac{\partial^{2} \phi}{\partial x^{2}}+\left(\frac{\partial \phi}{\partial x}\right)^{2}
$$

it follows that

$$
u_{1}=-\mathrm{i}\left(\frac{\partial w}{\partial x}+\frac{w}{\phi} \frac{\partial \phi}{\partial x}\right)
$$

solves (1.1). Note that correspondingly $\Phi=\mathrm{e}^{\phi}$ is a particular solution of (2.14).

\section{Mapping of potential systems to free particle systems}

By direct calculation one can prove the following theorem.

Theorem 1. Consider the Schrödinger equation (2.1). A point transformation maps a corresponding auxiliary potential system (2.13) into the free particle system

$$
\frac{\partial \hat{v}}{\partial y}=\mathrm{i} \hat{u} \quad \frac{\partial \hat{v}}{\partial \tau}=-\frac{\partial \hat{u}}{\partial y}
$$

for which each component $(\hat{u}(y, \tau), \hat{v}(y, \tau))$ satisfies the free particle equation if and only if the corresponding potential $V_{1}(x, t)$ is of the form

$$
V_{1}(x, t)=\frac{\mathrm{i} \partial \Psi / \partial t-\partial^{2} \Psi / \partial x^{2}}{\Psi}+2\left(\frac{\partial \Psi / \partial x}{\Psi}\right)^{2}=\hat{\alpha}(t) x^{2}+\hat{\beta}(t) x+\hat{\gamma}(t)-2 \frac{\partial^{2}}{\partial x^{2}} \log \Psi
$$

for arbitrary $\hat{\alpha}(t), \hat{\beta}(t), \hat{\gamma}(t)$, with $\Psi(x, t)$ satisfying

$$
\mathrm{i} \frac{\partial \Psi}{\partial t}+\frac{\partial^{2} \Psi}{\partial x^{2}}=\left[\hat{\alpha}(t) x^{2}+\hat{\beta}(t) x+\hat{\gamma}(t)\right] \Psi .
$$


(Note that $\Psi(x, t)$ satisfies (3.3) for any solution $\Phi(x, t)=1 / \Psi(x, t)$ of the corresponding adjoint equation of (3.3), which is the Schrödinger equation (2.14) with potential $\hat{\alpha}(t) x^{2}+$ $\hat{\beta}(t) x+\hat{\gamma}(t)$.) by

The corresponding mapping of system (2.13) to the free particle system (3.1) is given

$$
\begin{aligned}
& y=\sigma(t) x+\rho(t) \quad \tau=\int^{t} \sigma^{2}(\mu) \mathrm{d} \mu \\
& \hat{u}=\frac{1}{\sigma} \mathrm{e}^{\mathrm{i} g(x, t)}\left[u_{1}+\left\{\left(\frac{\dot{\sigma} x+\dot{\rho}}{2 \sigma}\right)-\mathrm{i} \frac{\partial \Psi / \partial x}{\Psi}\right\} \Psi v_{1}\right] \quad \hat{v}=\mathrm{e}^{\mathrm{i} g(x, t)} \Psi v_{1}
\end{aligned}
$$

where

$$
g(x, t)=\frac{\dot{\sigma}}{4 \sigma} x^{2}+\frac{\dot{\rho}}{2 \sigma} x+\lambda(t)
$$

and $(\sigma(t), \rho(t), \lambda(t))$ are related to $(\hat{\alpha}(t), \hat{\beta}(t), \hat{\gamma}(t))$ through the system of ordinary differential equations (ODEs)

$$
\hat{\alpha}(t)=\frac{\sigma \ddot{\sigma}-2 \dot{\sigma}^{2}}{4 \sigma^{2}} \quad \hat{\beta}(t)=\frac{\sigma \ddot{\rho}-2 \dot{\rho} \dot{\sigma}}{2 \sigma^{2}} \quad \hat{\gamma}(t)=\dot{\lambda}-\mathrm{i} \frac{\dot{\sigma}}{2 \sigma}-\frac{\dot{\rho}^{2}}{4 \sigma^{2}} .
$$

The transformation $S(t)=1 / \sigma(t)$ reduces the nonlinear system of ODEs (3.6) to a linear system. For details see the appendix in Bluman and Shtelen (1996).

Note that from (2.4) and (2.5) one can see that the point transformation

$$
y=\sigma(t) x+\rho(t) \quad \tau=\int^{t} \sigma^{2}(\mu) \mathrm{d} \mu \quad \hat{\Psi}=\mathrm{e}^{\mathrm{i} g(x, t)} \Psi
$$

maps equation (3.3) to the free particle equation

$$
i \frac{\partial \hat{\Psi}}{\partial \tau}+\frac{\partial^{2} \hat{\Psi}}{\partial y^{2}}=0
$$

where $g(x, t)$ is given by (3.5).

The previously known result concerning the equivalence under point transformations of Schrödinger equations with quadratic potentials (2.6) to a free particle equation immediately follows as a special case of theorem 1 .

If

$$
\frac{1}{\Psi} \frac{\partial \Psi}{\partial x}=\mathrm{i}\left[\frac{\dot{\sigma} x+\dot{\rho}}{2 \sigma}\right]
$$

then the non-local transformation (3.4) acting on $(x, t, u)$-space becomes a point transformation acting on $(x, t, u)$-space. Here equations (3.2) and (3.3) yield $V_{1}(x, t)=$ $\hat{\alpha}(t) x^{2}+\hat{\beta}(t) x+\hat{\gamma}(t)-\mathrm{i} \dot{\sigma} / \sigma$, where $\hat{\alpha}(t), \hat{\beta}(t), \hat{\gamma}(t)$ are given by (3.6). Then the substitution $\sigma \hat{u} \rightarrow u_{2}$ yields the point transformation mapping (2.1) to (2.3) with $V_{2}(y, \tau) \equiv 0$.

It is easy to see that the mapping (3.4) yields a non-local transformation of (2.1) to the free particle equation if and only if $\partial^{3} / \partial x^{3}(\log \Psi) \not \equiv 0$. Moreover, the resulting potential $V_{1}(x, t)$ is not of the quadratic form (2.6) if and only if

$$
\frac{\partial^{5}}{\partial x^{5}}(\log \Psi) \not \equiv 0
$$




\subsection{New potentials $V_{I}(x, t)$}

In general a solution $\Psi(x, t)$ of (3.3) leads to a complex potential $V_{1}(x, t)$ defined by (3.2). We must seek solutions $(\Psi(x, t), \hat{\alpha}(t), \hat{\beta}(t), \hat{\gamma}(t))$ of (3.3) so that $V_{1}(x, t)$ defined by (3.2) is a real function, and condition (3.9) is satisfied. Consequently it is obvious that $\hat{\alpha}(t)$ and $\hat{\beta}(t)$ must be real but $\hat{\gamma}(t)$ can be complex. Let

$$
\begin{aligned}
& \hat{\gamma}(t)=m(t)+\mathrm{i} c(t) \\
& \Psi(x, t)=A(x, t) \mathrm{e}^{\mathrm{i} \varphi(x, t)}
\end{aligned}
$$

where $m(t), c(t), A(x, t)$ and $\varphi(x, t)$ are real functions. If $V_{1}(x, t)$ defined by (3.2) is real then it is necessary that $2 \partial^{2} \varphi / \partial x^{2}=c(t)$, and hence

$$
\varphi(x, t)=\frac{1}{4} c(t) x^{2}+d(t) x+e(t)
$$

for some undetermined functions $c(t), d(t)$ and $e(t)$. Thus

$$
V_{1}(x, t)=\hat{\alpha}(t) x^{2}+\hat{\beta}(t) x+m(t)-2 \frac{\partial^{2}}{\partial x^{2}} \log A(x, t) .
$$

Now condition (3.9) becomes

$$
\frac{\partial^{5}}{\partial x^{5}} \log A \not \equiv 0 \text {. }
$$

After equating the real and imaginary parts of both sides of equation (3.3), we find that

$$
\begin{aligned}
& \frac{\partial A}{\partial t}+(c x+2 d) \frac{\partial A}{\partial x}=\frac{1}{2} c A \\
& \frac{\partial^{2} A}{\partial x^{2}}=\left[\left(\frac{1}{2} c x+d\right)^{2}+\frac{1}{4} \dot{c} x^{2}+\dot{d} x+\dot{e}+\hat{\alpha} x^{2}+\hat{\beta} x+m\right] A .
\end{aligned}
$$

From equation (3.15) we see that $A(x, t)$ is of the form

$$
A(x, t)=f(t) G(z)
$$

with

$$
z=r(t) x+s(t)
$$

for some functions $f(t), r(t)$ and $s(t)$. After substituting (3.17) into (3.15) and then into (3.16), we find that

$$
\begin{aligned}
& ([\dot{r}+c r] x+\dot{s}+2 d r) f G^{\prime}=\left(\frac{1}{2} c f-\dot{f}\right) G \\
& r^{2} G^{\prime \prime}=\left(\left[\frac{1}{2} c x+d\right]^{2}+\frac{1}{4} \dot{c} x^{2}+\dot{d} x+\dot{e}+\hat{\alpha} x^{2}+\hat{\beta} x+m\right) G
\end{aligned}
$$

where $G^{\prime}=\mathrm{d} G / \mathrm{d} z, G^{\prime \prime}=\mathrm{d}^{2} G / \mathrm{d} z^{2}$.

One can show that condition (3.14) and the compatibility conditions arising from equations (3.18)-(3.20), lead to $G(z)$ satisfying the differential equation

$$
G^{\prime \prime}=\left(M z^{2}+N z+P\right) G
$$

where $M, N$ and $P$ can be arbitrary constants, and after setting $\hat{k}=\hat{\alpha}-M r^{4}, \hat{\ell}=\hat{\beta}-$ $r^{3}(2 M s+N)$ and $\hat{m}=m-r^{2}\left(P+N s+M s^{2}\right)$, one finds that $\{c(t), d(t), e(t), f(t), r(t), s(t)\}$ satisfies the system of first-order differential equations

$$
\begin{aligned}
& \dot{c}+c^{2}=-4 \hat{k} \\
& \dot{d}+c d=-\hat{\ell} \\
& \dot{e}=-\hat{m}-d^{2} \\
& \dot{f}-\frac{1}{2} c f=0 \\
& \dot{r}+c r=0 \\
& \dot{s}=-2 d r .
\end{aligned}
$$


Inspecting equations $(3.22 a-f)$, we see that two cases arise.

Case I: $c(t) \not \equiv 0$. Here $\hat{\alpha}(t), \hat{\beta}(t)$ and $m(t)$ can be arbitrary functions. Once $c(t)$ is determined from $(3.22 a)$, then $(3.22 b-f)$ are linear differential equations and, in principle, can be solved in closed form. The Riccati transformation $c=\dot{C} / C$ transforms (3.22a) to

$$
\ddot{C}+4 \hat{k} C=0 .
$$

The general solution of (3.23) leads to the general solution of (3.22a-f) in closed form.

Case II: $c(t) \equiv 0$. Here $\hat{\beta}(t)$ and $m(t)$ can be arbitrary functions, but now $\hat{\alpha}$ is restricted to being an arbitrary constant, $\hat{\alpha}=\hat{\alpha}_{0}$. In this case, without loss of generality, equations $(3.22 a-f)$ reduce to

$$
\begin{array}{lcc}
r=1 & f=1 \quad \ddot{s}+4 \hat{\alpha}_{0} s=2(\hat{\beta}(t)-N) \\
d=-\frac{1}{2} \dot{s} & \dot{e}=-m(t)+P+N s+\hat{\alpha}_{0} s^{2}-\frac{1}{4} \dot{s}^{2} .
\end{array}
$$

Here the constant $M=\hat{\alpha}_{0}$ in differential equation (3.21).

In both cases the determination of $c(t), d(t)$ and $e(t)$ leads to the argument $\varphi(x, t)$ defined by equation (3.12); the determination of $f(t), r(t)$ and $s(t)$ leads to $A(x, t)$ given by equation (3.17) with $G(z)$ as any solution of the differential equation (3.21). Almost all solutions of (3.21) lead to $A(x, t)$ satisfying condition (3.14). Consequently, real, nonquadratic potentials $V_{1}(x, t)$ are obtained for which non-local transformations connect the free particle equation to the Schrödinger equation (2.1).

\subsection{Examples of new potentials $V_{l}(x, t)$}

As examples of new potentials $V_{1}(x, t)$ for which the Schrödinger equation (2.1) can be mapped into the free particle equation through our procedure, suppose $c(t)=0$, $\hat{\alpha}(t)=\hat{\alpha}_{0}=M=N=0, P=1$.

Here the solution of equations (3.21) and (3.24) leads to the class of time-dependent potentials

$$
V_{1}(x, t)=\hat{\beta}(t) x+m(t)-2 \operatorname{sech}^{2} z
$$

where $\hat{\beta}(t)$ and $m(t)$ are arbitrary functions, and

$$
z=x+\rho(t)
$$

with

$$
\rho(t)=2 \int^{t} \mathrm{~d} t_{2} \int^{t_{2}} \hat{\beta}\left(t_{1}\right) \mathrm{d} t_{1} .
$$

A corresponding mapping (3.4) of the Schrödinger equation (2.1) to the free particle equation has

$$
\begin{aligned}
& y=x+\rho(t) \quad \tau=t \\
& g(x, t)=\left[\int^{t} \hat{\beta}\left(t_{1}\right) \mathrm{d} t_{1}\right] x+\int^{t}\left[m\left(t_{2}\right)+\left[\int^{t_{2}} \hat{\beta}\left(t_{1}\right) \mathrm{d} t_{1}\right]^{2}\right] \mathrm{d} t_{2}, \\
& \sigma=1 \quad \frac{\dot{\rho}}{2 \sigma}=\int^{t} \hat{\beta}\left(t_{1}\right) \mathrm{d} t_{1} \\
& \Psi(x, t)=\cosh z \exp (\mathrm{i}[t-g(x, t)])
\end{aligned}
$$

with potential $V_{1}(x, t)$ given by (3.25) and $\rho(t)$ given by (3.27). 
Note that $V_{1}(x, t)$ has no singularities if $\hat{\beta}(t)$ and $m(t)$ are finite in $t$. Moreover, as $x \rightarrow \pm \infty$,

$$
V_{1}(x, t) \sim \hat{\beta}(t) x+m(t) .
$$

When $\hat{\beta}(t)=m(t)=0$, then $\rho(t)=0$, and

$$
V_{1}(x, t)=-2 \operatorname{sech}^{2} x
$$

which is a reflectionless time-independent potential discussed in section 2.4 of Dodd et al (1982).

\section{Concluding remarks}

The results presented in section 3 can be recycled to obtain further sets of Schrödinger equations with time-dependent potentials which can be transformed to the free particle equation by non-local transformations. Here the potentials $V_{1}(x, t)$ are given by equation (3.13) and the mapping formulae (3.4)-(3.7) relate the corresponding Schrödinger equations (2.1) to the free particle equation. Then (2.18) yields $V_{2}(x, t)$ with $\Phi_{1}(x, t)=$ $\Phi(x, t)$ defined by (2.14). In general, the factor $\Phi(x, t)=\Phi_{n}(x, t)$ satisfies (2.14), $w=u_{n}(x, t)$ satisfies $(2.17)$ and potential $V_{n+1}(x, t)$ is related to potential $V_{n}(x, t)$ through the corresponding generalization of (2.18), $n=1,2, \ldots$. The mapping formulae (3.4)-(3.7) are recycled accordingly. However, in general, the potentials $V_{n}(x, t)$ are complex for $n \geqslant 2$. One can show that for potentials $V_{2}(x, t)$ to be real, it is necessary that potentials $V_{1}(x, t)$ be restricted to those obtained from case II with $c(t)=0$.

When the potential $V_{1}(x, t)$ is time-independent, i.e. $V_{1}(x, t) \equiv V_{1}(x)$, one can show that the results obtained in the present paper can be deduced from the work exhibited in Bluman and Reid (1989) which is related to the factorization method introduced in the celebrated paper by Infeld and Hull (1951). In this case the recycling procedure always yields real time-independent potentials $V_{n}\left(x ; \alpha_{1}, \alpha_{1}, \ldots, \alpha_{2 n-2}\right)$ depending on $2 n-2$ arbitrary constants $\alpha_{1}, \alpha_{2}, \ldots, \alpha_{2 n-2}$.

For a complete discussion of the recycling procedure as it applies to mapping diffusion equations to the heat equation, see Bluman and Shtelen (1996).

\section{References}

Bluman G W 1980 SIAM J. Appl. Math. 39238

-1983 SIAM J. Appl. Math. 431259

Bluman G W and Doran-Wu P 1995 Acta Appl. Math. 4121

Bluman G W and Reid G J 1989 J. Math. Anal. Appl. 144565

Bluman G W and Shtelen V M 1996 On non-local transformations of diffusion processes into the Wiener process, submitted

Boyer C P 1974 Helv. Phys. Acta. 47589

Dodd R K, Eilbeck J C, Gibbon J D and Morris H C 1982 Solutions and Nonlinear Wave Equations (London: Academic)

Infeld L and Hull T E 1951 Rev. Mod. Phys. 2321

Niederer U 1973 Helv. Phys. Acta 46191 